\title{
Linear Recursive Automotive Target Tracking Filter for Advanced Collision Warning Systems
}

\author{
Seul-Ki Han ${ }^{1}$, Won-Sang Ra ${ }^{2, *}$, Ick-Ho Whang ${ }^{3}$ and Jin Bae Park ${ }^{1}$ \\ ${ }^{1}$ Department of Electrical and Electronic Engineering, Yonsei University, Seoul, Korea \\ ${ }^{2}$ School of Mechanical and Control Engineering, Handong Global University, Gyoungbuk, Korea \\ ${ }^{3}$ Department of Guidance and Control, Agency for Defense Development, Taejeon, Korea
}

Received: 27 May. 2013, Revised: 1 Oct. 2013, Accepted: 2 Oct. 2013

Published online: 1 May. 2014

\begin{abstract}
This paper proposes an improved automotive target tracking scheme using FMCW radar which is necessary for the advanced collision warning systems. Since there exist strong nonlinear relationships between the FMCW radar measurements and the target state, the target tracking and data association in dense road clutters have been recognized as a quite challenging problem. It is obvious that the use of accurate range rate measurement might be an excellent choice to improve both target tracking and clutter suppression performances. This motivates us to develop a novel linear recursive automotive target tracking filter based on the measurement conversion in the predicted line-of-sight (LOS) Cartesian coordinate system (PLCCS). Since the $x$ axis of the PLCCS is set by the predicted LOS vector from the host to the target, if the LOS prediction error is imperceptible, the range rate can be approximated to the $x$ component of the relative target velocity in PLCCS. Employing the PLCCS drastically reduces the complexity of the problem and allows us to solve it within the framework of linear recursive Kalman filtering. Through the simulations, the superiority of the proposed method is compared to the existing nonlinear automotive target tracking filters.
\end{abstract}

Keywords: FMCW Radar, Automotive Target Tracking, Data Association, Predicted Line-of-Sight Cartesian Coordinate System (PLCCS)

\section{Introduction}

Recently, the collision warning system (CWS) based on the automotive FMCW radar plays an important role in the next generation automobile with enhanced safety features $[1,2,3]$. The FMCW radar has a significant merit of robustness against the changeable weather conditions compared to other automotive sensors such as laser, vision, sonar and so on [4]. Moreover, it can provide relatively accurate target measurements even at long range. In this reason, the FMCW radar has compelled the attention of many engineers as an attractive solution for the CWS with high fidelity. Using the FMCW radar measurements, the CWS tracks target vehicles, classifies the treating ones, and makes decision for collision warning if necessary. Since the overall performance of the decision making depends on the accuracy of the target state estimates, the automotive radar target tracking becomes one of the core technologies for ensuring the reliability of the CWS $[5,6]$.
The automotive target tracking using FMCW radar is plagued by two difficulties. First, the automotive target tracking filter should be able to discriminate the target measurement from the clutters which can often be faced in the real-road driving conditions. In such heavy clutter environment, it is known that the performance of the conventional probabilistic data association filter (PDAF) is frequently degraded severely [7]. In order to find an efficient data association in dense clutter environment, we concentrated on the fact that most FMCW radars provide relatively accurate range rate measurements as well as range and line-of-sight (LOS) angle measurements. The use of range rate information could be an effective way to improve the tracking performance even in the dense road clutter environment [8]. However, at this point, the second difficulty in automotive target tracking may arise; one should devise a new filtering scheme which can effectively deal with the strong nonlinearity between the range rate information measured in polar coordinated

\footnotetext{
* Corresponding author e-mail: wonsang @ handong.edu
} 
system (PCS) and the relative target motion described in Cartesian coordinate system (CCS).

So far, many researchers have focused on the usefulness of the range rate information and tried to develop a performed automotive target tracking scheme using range rate measurement. To do this, the famous extended Kalman filter (EKF) has been attempted as a viable solution [9]. However, it involves a number of flaws in practice; poor convergence behavior under low SNR conditions and sensitiveness to the initial guess. Besides, these problems could be magnified when the range rate measurements are used for the automotive target tracking because the use of range rate measurements causes severe nonlinearity compared to the use of range or LOS measurements. To overcome the limitations of the EKF based automotive target tracking, the sequential filtering (SF) technique employing unscented Kalman filter (UKF) was proposed [10,11]. This approach comes from the idea that the UKF shows somewhat better estimation performance than the EKF for highly nonlinear systems. To reduce the inherent nonlinearity of the automotive target tracking problem caused by the use of range rate information, the SF method adopted the two stage filtering scheme. At the first stage, the linear Kalman filter processes the pseudo relative target position measurements obtained by converting the range and LOS angle measurements to the position measurement in CCS. Then, the additional UKF sequentially updates the state by using the range rate measurement to complement the first stage filtering results. However, the SF method could not meet our expectation because the performance improvement of $\mathrm{UKF}$ is restrictive in many cases. Moreover, due to the complicated filter structure of SF, it does not flee from the heavy computational burden.

In order to radically solve the above mentioned problems, this paper proposes a linear recursive automotive target tracking filter based on the measurement conversion in the predicted line-of-sight CCS (PLCCS) [12,13]. The PLCCS is introduced to eliminate the nonlinearity of the problem itself. The $x$ axis of the PLCCS coincides with the predicted target LOS vector defined by the a priori estimate of the tracking filter. Provided that the LOS prediction error is negligible, in PLCCS, the range rate measurement can be approximated to the $x$ component of the relative target velocity. Meanwhile, the target position measurement in PLCCS is calculated by using the range and LOS angle measurements hence the noise statistics of the target position measurement should be examined thoroughly. Fortunately, this task can be readily accomplished relying on the so-called average mean and variance calculation method [14]. Therefore, in PLCCS, the automotive target tracking problem can be readily solved within the framework of the linear Kalman filter. Since the proposed automotive target tracking filter uses the range rate information, it is expected that the proposed method drastically improves the tracking and data association
Table 1: Definitions of coordinate systems

\begin{tabular}{|c|c|}
\hline frame & Definition \\
\hline $\mathrm{C}$ & $\begin{array}{l}\text { Cartesian coordinate system }(\mathrm{CCS}) \\
\text { origin : center of host vehicle } \\
\vec{X}^{C}: \text { FMCW radar boresight direction } \\
\vec{Y}^{C}: \text { right direction on the horizontal plane }\end{array}$ \\
\hline $\mathrm{L}$ & $\begin{array}{l}\text { Line of sight CCS (LCCS) } \\
\vec{X}^{L}: \text { FMCW radar boresight direction } \\
\lambda: \text { right direction on the horizontal plane }\end{array}$ \\
\hline $\mathrm{P}$ & $\begin{array}{l}\text { Predicted LCCS (PLCCS) } \\
\vec{X}^{P}: \text { predicted LOS direction } \\
\bar{\lambda} \text { : predicted LOS angle calculated } \\
\quad \text { by using the } \text { a priori target position estimate }\end{array}$ \\
\hline
\end{tabular}

performance in cluttered environment. In addition, for its linear recursive structure, the proposed automotive target tracking filer can avoid the various practical issues raised in the existing nonlinear filters and it is preferable for realtime automotive applications. Through computer simulations for the typical automotive target engagement scenario in dense clutter, the proposed linear recursive automotive radar target tracking filter designed in PLCCS is demonstrated.

\section{Linear Automotive Target Tracking Filter in PLCCS}

\subsection{Nonlinear System Model in CCS}

Before preceding the automotive target tracking filter design, the coordinate systems used in this paper are summarized in Table 1. For the brevity, but without loss of generality, it is assumed that the automotive FMCW radar is mounted near the vehicle center. All angles are defined in clockwise direction. The relations among the coordinate systems are depicted in Figure 1.

In most typical the automotive target engagement scenarios, relative accelerations are negligible, hence the target relative motion could be described by the constant velocity model in CCS.

$$
\mathbf{x}_{k+1}^{C}=F \mathbf{x}_{k}^{C}+G \mathbf{w}_{k}^{C}
$$

where the relevant vector and matrices are defined as

$$
\begin{gathered}
\mathbf{x}^{C} \triangleq\left[\begin{array}{llll}
x^{C} & y^{C} & \dot{x}^{C} & \dot{y}^{C}
\end{array}\right]^{T}, \\
F \triangleq\left[\begin{array}{cccc}
1 & 0 & T_{s} & 0 \\
0 & 1 & 0 & T_{s} \\
0 & 0 & 1 & 0 \\
0 & 0 & 0 & 1
\end{array}\right], G \triangleq\left[\begin{array}{cccc}
T_{s}^{2} / 2 & 0 & T_{s} & 0 \\
0 & T_{s}^{2} / 2 & 0 & T_{s}
\end{array}\right]^{T} .
\end{gathered}
$$




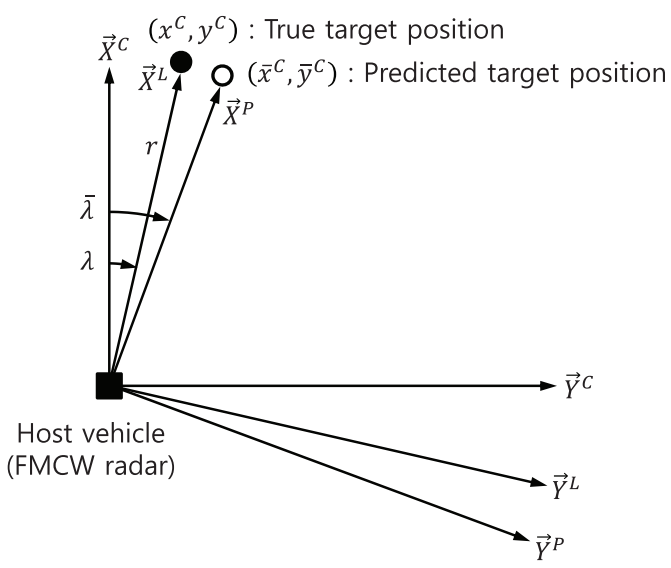

Fig. 1: Relationship between coordinate systems

In the above, the state vector $\mathbf{x}^{C}$ consists of the relative target position $\left(x^{C}, y^{C}\right)$ and velocity $\left(\dot{x}^{C}, \dot{y}^{C}\right) . T_{s}$ is sampling time. The process noise $\mathbf{w}^{C}$ is introduced to model the effect of relative target acceleration and is assumed to be zero-mean white Gaussian with covariance $Q^{C}$.

While the relative target motion is modeled in CCS, an automotive FMCW radar typically provides the range $r$, range rate $\dot{r}$, and LOS angle $\lambda$ which are the target information defined in PCS. In consideration of the measurement noises included in radar measurements, the measurement equation can be modeled by

$$
\mathbf{z}_{m, k}^{C}=\mathbf{z}_{k}^{C}+\mathbf{v}_{k}^{C}
$$

where

$$
\begin{gathered}
\mathbf{z}_{m}^{C}=\left[\begin{array}{c}
r_{m} \\
\lambda_{m} \\
\dot{r}_{m}
\end{array}\right], \mathbf{v}^{C}=\left[\begin{array}{c}
v_{r} \\
v_{\lambda} \\
v_{\dot{r}}
\end{array}\right], \\
\sqrt{\left(x^{C}\right)^{2}+\left(y^{C}\right)^{2}} \\
\mathbf{z}^{C}=\left[\begin{array}{c}
-1 \\
\left.\tan ^{C} / x^{C}\right) \\
\left(x^{C} \dot{x}^{C}+y^{C} \dot{y}^{C}\right) / \sqrt{\left(x^{C}\right)^{2}+\left(y^{C}\right)^{2}}
\end{array}\right] .
\end{gathered}
$$

In equation (2), $r_{m}, \dot{r}_{m}$ and $\lambda_{m}$ indicate the measured values of actual relative range, range rate and LOS angle, respectively. The uncorrelated noises $v_{r}, v_{\lambda}$, and $v_{\dot{r}}$ are assumed to be zero-mean white Gaussian with variance

$$
R_{k}^{C} \triangleq \operatorname{var}\left(\mathbf{v}_{k}^{C}\right)=\operatorname{diag}\left(\sigma_{r}^{2}, \sigma_{\lambda}^{2}, \sigma_{\dot{r}}^{2}\right) .
$$

As in (2), the true output vector $\mathbf{z}^{C}$ is expressed by the nonlinear function of the target state vector $\mathbf{x}^{C}$ in (1). Especially, the range rate information is highly nonlinear compared to the target position measurements $\left(r_{m}, \lambda_{m}\right)$ in PCS. This is one of the main reasons which make the automotive target tracking problem difficult. It is known that the range rate measurement is very effective for data association in dense clutter environment. However, the previous automotive target tracking schemes based on nonlinear state estimator could not properly handle the strong nonlinearity of the range rate and therefore we have much room for improvement. To avoid the increasing nonlinearity due to the use of range rate information, the automotive target tracking problem should be solved in the linear state estimation setting.

\subsection{Linear System Model in PLCCS}

The proposed linear automotive target tracking filter is devised based on the linear measurement equation in PLCCS. Apart from the existing approaches, the resultant linear measurement equation we will derive here contains the range rate information needed for the advanced data association techniques. Let us assume that the a priori target state vector $\overline{\mathbf{x}}^{C}$ in CCS is available from the automotive target tracking filter.

$$
\overline{\mathbf{x}}^{C}=\left[\begin{array}{llll}
\bar{x}^{C} & \bar{y}^{C} & \overline{\dot{x}}^{C} & \bar{y}^{C}
\end{array}\right]^{T}
$$

Using, $\overline{\mathbf{x}}^{C}$ the predicted LOS direction can be calculated, $\bar{\lambda}=\tan ^{-1}\left(\bar{y}^{C} / \bar{x}^{C}\right)$. Note that, at every time update procedure of the automotive target tracking filter, the PLCCS should also be updated. If the target position is predicted with considerable accuracy by the tracking filter within the sampling time $T_{s}$, the difference between true LOS angle $\lambda$ and the predicted LOS angle $\bar{\lambda}$ is negligible $(\lambda-\bar{\lambda} \approx 0, \dot{\lambda} \approx 0)$. Under this standing assumption, one can approximate the range rate $\dot{r}$ to the $x$ component of the relative target velocity in PLCCS.

$$
\dot{x}^{P} \approx \dot{r}
$$

It is very interesting that, if, instead of CCS, the PLCCS is adopted for automotive target tracking, the nonlinearity between the range rate information and the target state could be successfully eliminated as in (5). This implies that one can readily design the linear automotive target tracking filter in PLCCS.

Now, the FMCW radar measurement equation and the measurement noise statistics are rederived in PLCCS. To do this, let us consider the following matrices used for coordinate transform.

$$
\begin{gathered}
T_{3} \triangleq\left[\begin{array}{cc}
T_{2} & O_{2 \times 1} \\
O_{1 \times 2} & 1
\end{array}\right], T_{4} \triangleq\left[\begin{array}{cc}
T_{2} & O_{2 \times 2} \\
O_{2 \times 2} & T_{2}
\end{array}\right], \\
T_{2} \triangleq\left[\begin{array}{cc}
\cos (\bar{\lambda}) & \sin (\bar{\lambda}) \\
-\sin (\bar{\lambda}) & \cos (\bar{\lambda})
\end{array}\right]
\end{gathered}
$$

It should be pointed out that $T_{3}$ and $T_{4}$ are computed using the predicted LOS $\bar{\lambda}$ or the function of a priori target position estimate such as $\tan ^{-1}\left(y^{C} / x^{C}\right)$. With defining $\mathbf{x}^{P}$ be the target state vector in PLCCS, the following equation holds.

$$
\mathbf{x}^{P}=T_{4} \mathbf{x}^{C}
$$


For converting the radar measurements $\mathbf{z}_{m}^{C}$ (2) to the measurements in PLCCS, let us consider the following relation.

$$
\begin{aligned}
\zeta_{m}^{C} & \triangleq\left[\begin{array}{c}
r_{m} \cos \left(\lambda_{m}\right) \\
r_{m} \sin \left(\lambda_{m}\right) \\
\dot{r}_{m}
\end{array}\right] \\
& =\left[\begin{array}{c}
x_{m}^{C} \\
y_{m}^{C} \\
\dot{r}_{m}
\end{array}\right]=\left[\begin{array}{c}
x^{C} \\
y^{C} \\
\dot{r}
\end{array}\right]+\left[\begin{array}{c}
v_{x}^{C} \\
v_{y}^{C} \\
v_{\dot{r}}
\end{array}\right]=\zeta^{C}+\mathbf{v}^{C}
\end{aligned}
$$

In the above $\zeta^{C}$ denotes the true target position and range rate in $\mathrm{CCS} . \mathbf{v}^{C}$ means the pseudo-position and range rate measurement errors. Based on the approximation (5), the PLCCS measurement equation (9) is obtained by applying the coordinate transformation matrix $T_{3}$ to the pseudo measurement equation (8).

$$
\zeta_{b, m}^{P}=T_{3} \zeta_{m}^{C} \approx H \mathbf{x}^{P}+\mathbf{v}^{P}
$$

where

$$
H \triangleq\left[\begin{array}{llll}
1 & 0 & 0 & 0 \\
0 & 1 & 0 & 0 \\
0 & 0 & 1 & 0
\end{array}\right], \quad \mathbf{v}^{P} \triangleq\left[\begin{array}{c}
r_{m} \cos \left(\lambda_{m}-\bar{\lambda}\right)-r \cos (\lambda-\bar{\lambda}) \\
r_{m} \sin \left(\lambda_{m}-\bar{\lambda}\right)-r \sin (\lambda-\bar{\lambda}) \\
\dot{r}_{m}-\dot{r}
\end{array}\right]
$$

In the above equation, $\mathbf{x}^{P}$ and $\mathbf{v}^{P}$ are the true target state vector in PLCCS and the PLCCS measurement error, respectively.

Note that, different from the nonlinear measurement equation (2), the automotive target tracking problem in PLCCS is related to the linear measurement equation (9). Since $\mathbf{v}^{P}$ is expressed as a function of the FMCW radar measurements, $r_{m}, \dot{r}_{m}$, and $\lambda_{m}$ corrupted by the white Gaussian noises, it has complicated statistics. Using the concept of average true bias and covariance, the bias $\mathbf{b}^{P}$ and covariance $R^{P}$ of measurement error $\mathbf{v}^{P}$ are calculated as follows [14]:

$$
\begin{gathered}
\mathbf{b}^{P} \triangleq\left[\begin{array}{c}
r_{m} \cos \left(\lambda_{m}-\bar{\lambda}\right)\left(e^{-\sigma_{\lambda}^{2}}-e^{-\sigma_{\lambda}^{2} / 2}\right) \\
r_{m} \sin \left(\lambda_{m}-\bar{\lambda}\right)\left(e^{-\sigma_{\lambda}^{2}}-e^{-\sigma_{\lambda}^{2} / 2}\right)
\end{array}\right], \\
R^{P} \triangleq\left[\begin{array}{ccc}
R_{11}^{P} & R_{12}^{P} & 0 \\
R_{12}^{P} & R_{22}^{P} & 0 \\
0 & 0 & 0
\end{array}\right]
\end{gathered}
$$

where

$R_{11}^{P}=r_{m}^{2} e^{-2 \sigma_{\lambda}^{2}} \cdot \Delta_{1}+\sigma_{r}^{2} e^{-2 \sigma_{\lambda}^{2}} \cdot \Delta_{2}$

$R_{12}^{P}=s(\Delta \lambda) c(\Delta \lambda) e^{-4 \sigma_{\lambda}^{2}}\left[\sigma_{r}^{2}+\left(r_{m}^{2}+\sigma_{r}^{2}\right)\left(1-e^{\sigma_{\lambda}^{2}}\right)\right]$

$R_{22}^{P}=r_{m}^{2} e^{-2 \sigma_{\lambda}^{2}} \cdot \Delta_{3}+\sigma_{r}^{2} e^{-2 \sigma_{\lambda}^{2}} \cdot \Delta_{4}$

$\Delta_{1}=c^{2}(\Delta \lambda)\left(\operatorname{ch}\left(2 \sigma_{\lambda}^{2}\right)-\operatorname{ch}\left(\sigma_{\lambda}^{2}\right)\right)+s^{2}(\Delta \lambda)\left(\operatorname{sh}\left(2 \sigma_{\lambda}^{2}\right)-\operatorname{sh}\left(\sigma_{\lambda}^{2}\right)\right)$

$\Delta_{2}=c^{2}(\Delta \lambda)\left(2 \operatorname{ch}\left(2 \sigma_{\lambda}^{2}\right)-\operatorname{ch}\left(\sigma_{\lambda}^{2}\right)\right)+s^{2}(\Delta \lambda)\left(2 \operatorname{sh}\left(2 \sigma_{\lambda}^{2}\right)-\operatorname{sh}\left(\sigma_{\lambda}^{2}\right)\right)$

$\Delta_{3}=s^{2}(\Delta \lambda)\left(\operatorname{ch}\left(2 \sigma_{\lambda}^{2}\right)-\operatorname{ch}\left(\sigma_{\lambda}^{2}\right)\right)+c^{2}(\Delta \lambda)\left(\operatorname{sh}\left(2 \sigma_{\lambda}^{2}\right)-\operatorname{sh}\left(\sigma_{\lambda}^{2}\right)\right)$

$\Delta_{4}=s^{2}(\Delta \lambda)\left(2 \operatorname{ch}\left(2 \sigma_{\lambda}^{2}\right)-\operatorname{ch}\left(\sigma_{\lambda}^{2}\right)\right)+c^{2}(\Delta \lambda)\left(2 \operatorname{sh}\left(2 \sigma_{\lambda}^{2}\right)-\operatorname{sh}\left(\sigma_{\lambda}^{2}\right)\right)$

In the above equation, $c$ and $s$ are used as $\cos$ and $\sin$. $c h$ and $s h$ mean $\cosh$ and $\sinh$, respectively. $\Delta \lambda$ denotes $\lambda_{m}-\bar{\lambda}$. Subtracting the bias $\mathbf{b}^{P}$ in both sides of (9), one gets the resultant linear measurement equation in PLCCS.

$$
\zeta_{m}^{P} \triangleq \zeta_{b, m}^{P}-\mathbf{b}^{P}=T_{3} \zeta_{m}^{C}-b^{P}
$$

\subsection{Linear Target Tracking Filter in PLCCS}

In this subsection, to cope with the clutter environment, the conventional PDAF is slightly modified for using the linear measurement equation derived in PLCCS. It can prevent the performance degradation due to the strong nonlinearity of range rate information and cluttered measurements. The detailed description for the PLCCS based PDAF algorithm is summarized as follows:

\section{Step 1. Time update: predicted estimate}

The time update of the target state and the corresponding error covariance matrix is conducted in CCS.

$$
\overline{\mathbf{x}}_{k}^{C}=F \hat{\mathbf{x}}_{k-1}^{C}, \quad \bar{P}_{k}^{C}=F \hat{P}_{k-1}^{C} F^{T}+Q_{k}^{C}
$$

\section{Step 2. Calculation of coordinate transformation matrices}

Once the predicted LOS $\bar{\lambda}_{k}=\tan ^{-1}\left(\bar{y}_{k}^{C} / \bar{x}_{k}^{C}\right)$ is calculated by using the a priori estimate $\overline{\mathbf{x}}_{k}^{C}$, the transformation matrices $T_{2, k}, T_{3, k}$, and $T_{4, k}$ are computed by using the definitions of (6).

\section{Step 3. CCS to PLCCS coordinate transform}

Using the transformation matrices, the a priori estimate, the error covariance matrix, and the radar measurements are converted to PLCCS. Since the measurement bias $\mathbf{b}_{k}^{P}(i)$ is generated by this coordinate transform procedure, the bias compensation technique is applied as shown in (11).

$$
\begin{gathered}
\overline{\mathbf{x}}_{k}^{P}=T_{4, k} \overline{\mathbf{x}}_{k}^{C}, \bar{P}_{k}^{P}=T_{4, k} \bar{P}_{k}^{C} T_{4, k}^{T} \\
\zeta_{m, k}^{P}(i)=T_{3, k} \zeta_{m, k}^{C}(i)-\mathbf{b}_{k}^{P}(i), \quad i=1, \ldots, M_{k}
\end{gathered}
$$

where $M_{k}$ is the number of measurements originated from the target and clutters.

The residual vector $e_{k}^{P}$ and its covariance $S_{k}^{P}$ are computed using the a priori estimate and PLCCS measurement.

$$
\begin{aligned}
& \mathbf{e}_{k}^{P}(i)=\zeta_{m, k}^{P}(i)-H \overline{\mathbf{x}}_{k}^{P}, \quad i=1, \ldots, M_{k} \\
& S_{k}^{P}(i)=H \bar{P}_{k}^{P} H^{T}+R_{k}^{P}(i)
\end{aligned}
$$

where $R_{k}^{P}(i)$ is defined in (11).

\section{Step 4. Measurement validation}

For generating data-association hypotheses for PDAF, the radar measurements within the following validation region are considered.

$$
\left(\mathbf{e}_{k}^{P}(i)\right)^{T}\left(S_{k}^{P}(i)\right)^{-1} \mathbf{e}_{k}^{P}(i) \leq \gamma
$$

In the above, $\gamma$ is the threshold for measurement validation. The residue is Gaussian distributed; hence the left side of 
(15) has the chi-square distribution. Considering its degree of freedom, $\gamma=11.34$ is chosen for our application. The volume of validation region is given by

$$
V_{k}=c_{n_{z}} \gamma^{n_{z} / 2}\left|S_{k}^{P}(i)\right|^{1 / 2}
$$

where $n_{z}$ is the dimension of measurement vector and $c_{n_{z}}$ is decided depending on $n_{z}$. In our case, $n_{z}=3, c_{n_{z}}=4 \pi / 3$.

Step 5. Measurement update and data association probability calculation

The measurement update using validated measurements is carried out as follows:

$$
\begin{aligned}
& \hat{\mathbf{x}}_{k}^{P}(i)= \begin{cases}\overline{\mathbf{x}}_{k}^{P}+K_{k}^{P} \mathbf{e}_{k}^{P}(i), & i=1, \ldots, m_{k} \\
\overline{\mathbf{x}}_{k}^{P}, & i=0\end{cases} \\
& \hat{P}_{k}^{P}(i)= \begin{cases}\left(I_{4 \times 4}-K_{k}^{P}(i) H\right) \bar{P}_{k}^{P}, & i=1, \ldots, m_{k} \\
\bar{P}_{k}^{P}, & i=0\end{cases}
\end{aligned}
$$

where

$$
K_{k}^{P}(i)=\bar{P}_{k}^{P} H^{T}\left(S_{k}^{P}(i)\right)^{-1}
$$

In the above equation, $i=0$ means that there is no validated measurement originated from the target. $m_{k}$ is the total number of validated measurements.

The probability for testing the reliability of each data association hypothesis is given by

$$
\beta_{k}(i)= \begin{cases}c e^{-1 / 2\left(\mathbf{e}_{k}^{P}(i)\right)^{T}\left(S_{k}^{P}(i)\right)^{-1} \mathbf{e}_{k}^{P}(i)}, & i=1, \ldots, m_{k} \\ c\left|2 \pi S_{k}^{P}(i)\right|^{1 / 2} m_{k} \frac{1-P_{G} P_{D}}{V_{k} P_{D}}, & i=0\end{cases}
$$

where $P_{D}$ is target detection probability and $P_{G}$ is the probability with which the target measurement falls in the validation region.

\section{Step 6. Gaussian mixtured estimate and estimation error covariance}

The a posteriori target state estimate of the proposed PDAF and its error covariance are defined under the same concept of the conventional PDAF.

$$
\begin{aligned}
& \hat{\mathbf{x}}_{k}^{P}=\sum_{i=0}^{m_{k}} \hat{\mathbf{x}}_{k}^{P}(i) \beta_{k}(i) \\
& \hat{P}_{k}^{P}=\sum_{i=0}^{m_{k}}\left[\hat{P}_{k}^{P}(i)+\left(\hat{\mathbf{x}}_{k}^{P}(i)-\hat{\mathbf{x}}_{k}^{P}\right)\left(\hat{\mathbf{x}}_{k}^{P}(i)-\hat{\mathbf{x}}_{k}^{P}\right)^{T}\right] \beta_{k}(i)
\end{aligned}
$$

\section{Step 7. PLCCS to CCS coordinate transform: filtered estimate}

Finally, the target state estimate and error covariance matrix in PLCCS are converted to CCS.

$$
\hat{\mathbf{x}}_{k}^{C}=T_{4, k}^{T} \hat{\mathbf{x}}_{k}^{P}, \quad \hat{P}_{k}^{C}=T_{4, k}^{T} \hat{P}_{k}^{P} T_{4, k}
$$

\section{Simulation Results}

Using a typical automotive target engagement scenario, the performance of the proposed linear target tracker is compared with existing nonlinear filters; EKF and SF based PDAFs. The simulation results of the optimal

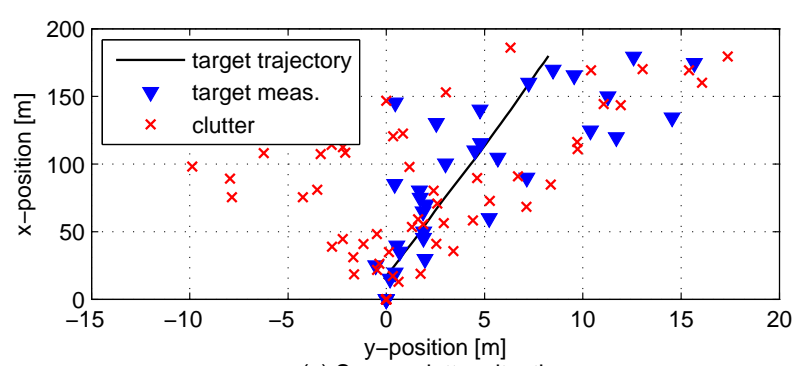

(a) Sparse clutter situation

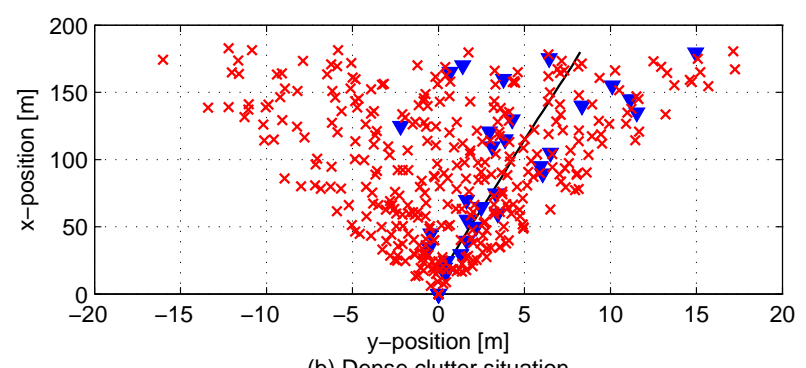

(b) Dense clutter situation

Fig. 2: Target trajectory and radar measurements

Kalman filter (OKF) which uses the correct data association. The OKF is not practically realizable because the correct data association is not available in actual applications, but it can be regarded as a reference of the best performance because it runs based on the ideally best data association. In order to compare the tracking performance in various clutter environments, the sparse and dense road clutter cases shown in Figure 2 are taken into account. Referring to the specifications for the commercial automotive FMCW radar ARS300 manufactured by Continental, the simulation conditions are set as follows:

$$
\sigma_{r}=0.25[m], \sigma_{\lambda}=1.5[\mathrm{deg}], \sigma_{\dot{r}}=0.14[\mathrm{~m} / \mathrm{s}], T_{s}=0.3[\mathrm{~s}]
$$

The root mean squared errors (RMSEs) of $x$ position and velocity estimates obtained from the 500 Monte Carlo trials are shown in Figure 3, 4. As expected, it seems that the position estimate of EKF based PDAF tends to diverge in cluttered environment. The SF based algorithm shows acceptable convergence property in the sparse road clutter condition since it can make more effective use of the range rate information for data association than EKF based tracking filter. However, its performance enhancement is restrictive in the dense clutter condition. The reason is why the SF still adopts the nonlinear filter structure and suffers from the side-effects caused by the intrinsic nonlinearity of the range rate information. On the contrary, regardless of clutter density, the proposed linear automotive target tracking filter shows reliable and satisfactory tracking performance similar to the OKF. This is because the proposed filter adopts the linear recursive structure which can accommodate the range rate measurement in natural way. 


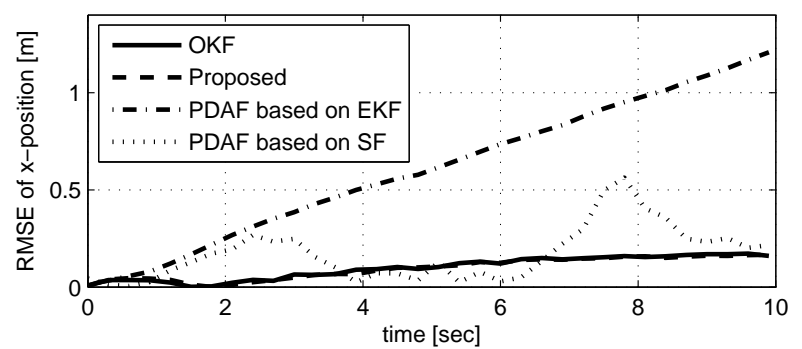

(a) Sparse clutter situation

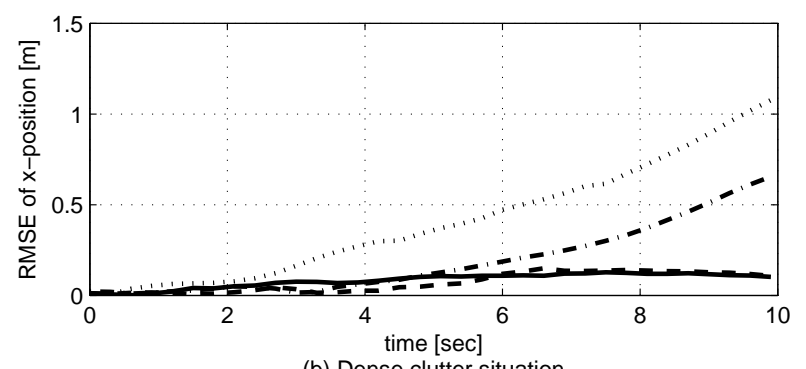

(b) Dense clutter situation

Fig. 3: RMSEs of $x$ position estimates

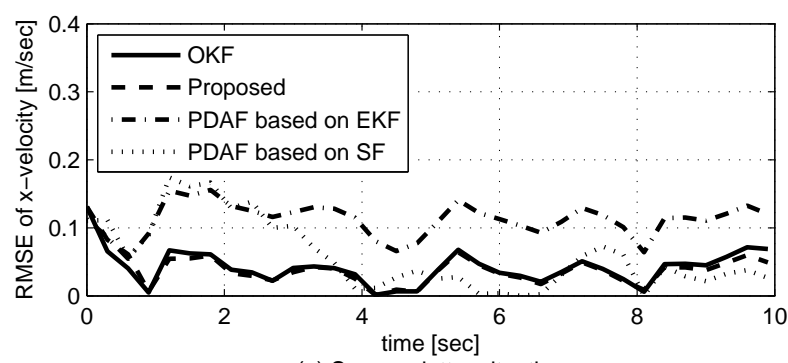

(a) Sparse clutter situation

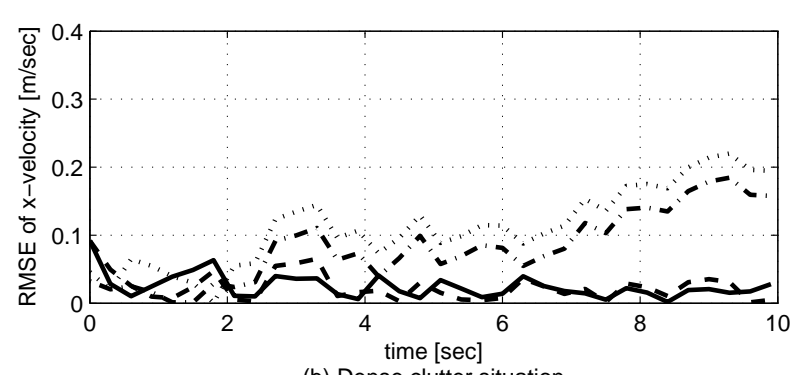

(b) Dense clutter situation

Fig. 4: RMSEs of $x$ velocity estimates

\section{Conclusion}

In this paper, a novel linear target tracking filter for automotive FMCW radar based CWS was proposed. To effectively cope with the problem caused by the high nonlinearity in range rate measurement and to improve the performance of tracking in clutter environment, the linear measurement equation has been newly derived in
PLCCS whose $x$ axis coincides with the predicted LOS vector from the host to the target vehicle. Hence, under the assumption that the target prediction error is small enough, the range rate measurement could be approximated to the $x$ component of relative target velocity measurement. Based on this practical observation, it was shown that the automotive target tracking problem results in to the linear filtering problem in PLCCS. Through the simulation results, the improved target tracking performance of the proposed filter was demonstrated. For its simple linear recursive structure, the suggested algorithm is preferable for various real-time automotive target tracking applications often encountered in dense road clutter environments.

\section{Acknowledgement}

This research was supported by Basic Science Research Program through the National Research Foundation of Korea (NRF) funded by the Ministry of Education (NRF-2010-0004224)

\section{References}

[1] P. E. An and C. J. Harris, IEEE Transactions on Systems, Man, and Cybernetics - Part A: Systems and Humans, 26, 1083-4427 (1996).

[2] A. Vahidi and A. Eskandarian, IEEE Transactions on Intelligent Transportation Systems, 4, 143-153 (2003).

[3] S. K. Gehrig and F. J. Stein, IEEE Transactions on Intelligent Transportation Systems, 8, 233-244 (2007).

[4] M. Mitsumoto, N. Uehara, S. Inatsune, and T. Kirimoto, IEICE Transactions on Communications, E83-B, 19831989 (2000).

[5] C. E. Smith, C. A. Richards, S. A. Brandt and N. P. Papanikolopoulos, IEEE Transactions on Vehicular Technology, 45, 744-759 (1996).

[6] P. Setlur, J. R. Wagner, D. M. Dawson and D. Braganza, IEEE Transactions on Vehicular Technology, 55, 76-85 (2006).

[7] S. S. Ahmeda, I. Harrison, M. S. Woolfson, IEE Proceedings Radar, Sonar and Navigation, 143, 17-22 (1996).

[8] J. Ru, H. Chen, X. R. Li, and G. Chen, Proceedings of the SPIE, 5913, 59131Q-1-59131Q-13 (2005).

[9] K. S. Miller and D. M. Leskiw, IEEE Transactions on Aerospace and Electronic Systems, AES-18, 192-200 (1982).

[10] Z. Duan, X. R. Li, C. Han, and H. Zhu, Proceedings of International Conference on Information Fusion, 130-137 (2005).

[11] M. Lei and C. Han, IEEE Transactions on Aerospace and Electronics Systems, 43, 239-250 (2007).

[12] S. T. Park and J. G. Lee, IEEE Transactions on Aerospace and Electronic Systems, 34, 1337-1344 (1998).

[13] F. Daum and R. Fitzgerald, IEEE Transactions on Automatic Control, 28, 269-283 (1983).

[14] D. Lerro and Y. Bar-Shalom, IEEE Transactions on Aerospace and Electronic Systems, 29, 1015-1022 (1993). 


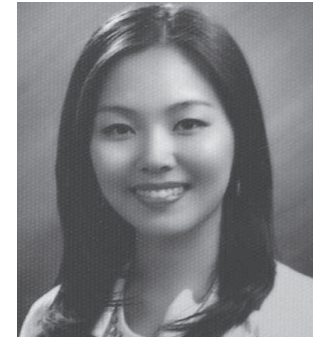

Seul-Ki Han received the B.E. degree in electrical and electronic engineering from Yonsei University, Seoul, Korea, in 2009, where she is currently working toward the Ph.D. degree. Her research interests include filtering theory, multiple target tracking, association filter and applications to FMCW radar.

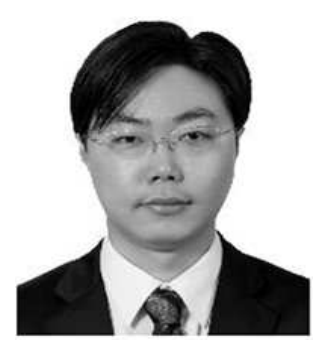

Won-Sang Ra received his B.E. degree in Electrical Engineering and his M.S. degree in Electrical and Computer Engineering, as well as his $\mathrm{Ph} . \mathrm{D}$. degree in Electrical and Electronics Engineering from Yonsei University, Seoul, Korea, in 1998, 2000, and 2009, respectively. From March 2000 to February 2009, he was with the Guidance and Control Department of the Agency for Defense Development, Daejeon, as a Senior Researcher. Since March 2009, he has been with the School of Mechanical and Control Engineering, Handong Global University, where he is currently an Assistant Professor. His main research topic includes robust filtering theory and its applications to autonomous vehicle guidance and control.

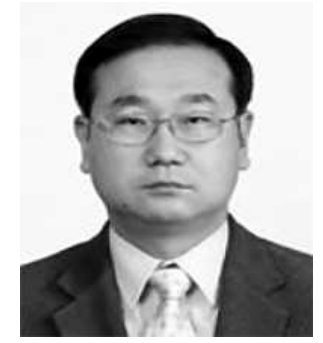

Ick-Ho Whang received his B.S., M.S. and Ph.D. degrees in Control and Instrumentation Engineering from Seoul National University, Seoul, Korea, in 1988, 1990, and 1995, respectively. He worked with the Department of Mechanical and Aerospace Engineering at the Naval Postgraduate School as a Visiting Researcher from 2003 to 2004. Since 1995, he has been with the Guidance and Control Department of the Agency for Defense Development, Daejeon, as a Principal Researcher. His research interest includes optimal filtering, target tracking and missile guidance.

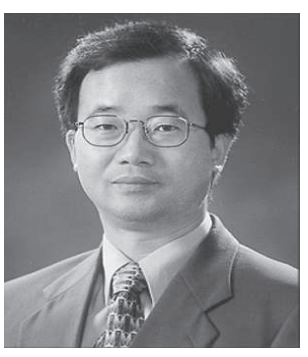

Jin Bae Park received the B.E. degree in electrical engineering from Yonsei University, Seoul, Korea, in 1977, and the M.S. and Ph.D. degrees in electrical engineering from Kansas State University, Manhattan, in 1985 and 1990, respectively. Since 1992, he has been with the Department of Electrical and Electronic Engineering, Yonsei University, where he is currently a Professor. His research interest includes robust control and filtering, nonlinear control, mobile robot, fuzzy logic control, neural networks, genetic algorithms, and Hadamardtransform spectroscopy. Dr. Park has served as the Director for the Transactions of the Korean Institute of Electrical Engineers (1998.2003) and the Institute of Control, Automation, and Systems Engineers (1999.2003). He is currently the Editorin- Chief for the International Journal of Control, Automation, and Systems. 